\title{
Relationship between Obesity and Korean and Mediterranean Dietary Patterns: A Review of the Literature
}

\author{
Eunhee Choi', Seong-Ah Kim², Hyojee Joung ${ }^{1,2, *}$ \\ 'Department of Public Health, Graduate School of Public Health and ${ }^{2}$ Institute of Health and Environment, Seoul National University, Seoul, Korea
}

Obesity is a well-known risk factor for various chronic diseases. Among the various risk factors for obesity, diet is one of the most modifiable. This study aimed to review current research on the association between obesity and the Korean diet compared with the Mediterranean diet. An electronic literature search was conducted using Medline and Embase. Totals of 10 and 17 studies were included in this review for the Korean dietary pattern (KDP) and the Mediterranean dietary pattern (MDP), respectively. These studies mainly applied factor analysis for the KDP and index analysis for the MDP. Only one of $10 \mathrm{KDP}$ articles reported a significant inverse association with obesity, while most MDP studies showed a preventive association with obesity. There are a limited number of KDP studies compared with MDP studies, and the methods of deriving the dietary patterns are different between the KDP and MDP. To produce more conclusive evidence on the association between the KDP and obesity, well-designed and controlled trials or large prospective cohort studies are needed.

Key words: Diet, Obesity, Mediterranean diet, Review

\author{
Received February 8, 2019 \\ Reviewed February 28, 2019 \\ Accepted March 11, 2019 \\ *Corresponding author \\ Hyojee Joung \\ https://orcid.org/0000-0003-1182-7786 \\ Department of Public Health, Graduate \\ School of Public Health, Seoul National \\ University, 1 Gwanak-ro, Gwanak-gu, \\ Seoul 08826, Korea \\ Tel: +82-2-880-2716 \\ Fax: +82-2-883-2832 \\ E-mail: hjjoung@snu.ac.kr
}

\section{INTRODUCTION}

Obesity is a well-known risk factor for various chronic diseases including cardiovascular diseases, type 2 diabetes mellitus (T2DM), hypertension, dyslipidemia, and some types of cancer. ${ }^{1-3}$ In Korea, the mortality rate due to cancer and cardiovascular disease and the prevalence of dyslipidemia have been steadily increasing concurrent with an increase in obesity. ${ }^{4,5}$

Globally, approximately 39\% adults aged 18 years and over were overweight in 2016, and $13 \%$ were obese. ${ }^{6}$ Likewise, the prevalence of obesity in Korea has increased steadily. Obesity in Korean adults increased from $26.0 \%$ in 1998 to $34.8 \%$ in $2016^{4}$, based on the Korea National Health and Nutrition Examination Survey.

Obesity has been described as multi-factorial, affected by both genetic and environmental factors including diet, physical activity, alcohol consumption, and psychosocial aspects. ${ }^{7,8}$ Among the various risk factors of obesity, diet is one of the most modifiable. ${ }^{9}$ The Dietary Guidelines for Americans 2015-2020 recommend increasing consumption of fruits, vegetables, whole grains, and fat-free or low-fat milk and milk products and limiting saturated fats, trans fats, cholesterol, salt (sodium), and added sugars to prevent weight gain and maintain healthy weight. ${ }^{10}$ When these individual nutrients are combined as a certain dietary pattern, its effect on health will be more than that of the individual nutrients. ${ }^{10} \mathrm{~A}$ dietary pattern is a broader picture of the overall diet rather than a single nutrient or food. It is more predictive when examining disease risk because it allows for complicated interactional and synergistic effects of nutrients and foods. ${ }^{10}$

Copyright ( 2019 Korean Society for the Study of Obesity

(a) This is an Open Access article distributed under the terms of the Creative Commons Attribution Non-Commercial License (http://creativecommons.org/licenses/by-nc/4.0/) which permits unrestricted non-commercial use, distribution, and reproduction in any medium, provided the original work is properly cited. 
With these advantages, numerous researchers have recently applied dietary pattern approaches in nutritional epidemiological studies. There has been extensive research on the associations between various dietary patterns - including the Mediterranean $\operatorname{diet}^{11-13}$, the Dietary Approaches to Stop Hypertension trial ${ }^{14,15}$, and the adherence to the Healthy Eating Index ${ }^{16}$ — and the risks of related chronic diseases including cancer, T2DM, and cardiovascular diseases. ${ }^{12,17}$ However, few studies have examined the effects of the Korean dietary pattern (KDP) on obesity, and their results have been inconsistent and inconclusive. ${ }^{9,18-22}$ Conversely, one of the best-known healthy dietary patterns, the Mediterranean dietary pattern (MDP), has been extensively researched and is highly recommended. ${ }^{23-39}$ Emerging research suggests that the health benefits of this diet go beyond obesity and include reduced risk of various chronic diseases. ${ }^{11-13}$ Before advocating specific dietary patterns to promote health and prevent diseases, it is necessary to evaluate the existing scientific evidence and draw a comprehensive conclusion. Therefore, this study aimed to review the current research on the association between obesity and the KDP compared with the MDP.

\section{METHODS}

An electronic literature search was conducted using Medline and Embase from November 23 to December 27, 2018. The range of publication years in the searched references was 2006 to 2018. The search terms were "obesity," "overweight," "weight gain," and "dietary pattern." The terms "Korea," "Korean," and "Mediterranean” were added depending on the target population. The reference lists of selected articles were also assessed.

The studies were eligible for inclusion in this review if they (1) explored association of dietary patterns and obesity rather than individual nutrients, (2) reported odds ratios (ORs) or hazard ratio as risk estimates, and (3) were in English. The articles were excluded if they (1) reported the risk estimates of regression coefficient and descriptive information, (2) had dependent variables of weight gain and loss (kg), fat-free mass, waist circumference, etc., or (3) were not in English. The complete search strategy is presented in Fig. 1.

\section{CHARACTERISTICS OF THE KDP}

Definition of the KDP differs depending on study and researchers. According to the Korea Food Research Institute, it is defined as "diets prepared by using Korean traditional cooking materials or similar food materials and made by own unique cooking method or similar method of Korea, and developed and handed down with the historical and cultural characteristics of Korean people." ${ }^{40}$ The Rural Development Administration defined the Korean diet as "a unique food or meal that Koreans have consumed for thousands of years." ${ }^{41}$ Although nutritional transition to Western dietary patterns (WDP), characterized by high consumption of fat and meat, has accelerated, the traditional Korean diet has maintained its aspects in distinct ways. ${ }^{42,43}$

Based on the literature, the traditional Korean diet is characterized by predominant consumption of plant-derived products and moderate intake of animal-derived food; it consists of grains (mainly rice), vegetables, seaweed, fish, and meat in moderation and thus has high carbohydrate and low fat contents. ${ }^{44}$ Particularly, rice is a staple food and forms the basis of the Korean diet. Extensive use of fermented foods such as kanjang, doenjang, cheonggukjang, gochujang, and $\mathrm{kimchi}^{45}$, which are known for their health benefits relating to antioxidant and anti-inflammatory activities ${ }^{46,47}$, is another characteristic of the Korean diet.

\section{CHARACTERISTICS OF THE MDP}

The concept of the Mediterranean diet was introduced in the early 1960s as the typical dietary pattern of Crete, much of the rest of Greece, and southern Italy. It was first publicized in 1975 by Keys $^{48}$ in a seven-country study and became widely recognized in the 1990s.

The Mediterranean diet is traditionally a collection of eating habits followed by people from the various countries in contact with the Mediterranean Sea, rather than a specific diet. ${ }^{49}$ According to a study by EL Rhazi et al. ${ }^{23}$, general components of the traditional Mediterranean diet are: (1) high consumption of plant foods (fruits, vegetables, breads, cereals, beans, nut, and seeds), (2) olive oil as the main source of added fat, (3) nonexcessive consumption of wine, (4) low to adequate consumption of cheese, yogurt, fish, 
poultry, and eggs, and (5) low intake of red and processed meat. However, it is difficult to define one particular MDP due to the diversity in Mediterranean countries. For example, a characteristic of the Italian MDP is high consumption of pasta, but in Spain, fish consumption is exceptionally high. In Greece, mainly whole grain bread and large amounts of vegetables and legumes are consumed. ${ }^{24}$

To identify extent of adherence to the MDP, the Mediterranean diet score (MDS) was created. Various scoring systems for MDP have been developed and applied in different cohort studies. In the most widely used scoring system developed by Trichopoulou et al. ${ }^{50}$ in 2003, using the sex-specific median as the cutoff, a value of 0 or 1 is allocated to each of nine components. For beneficial components, including vegetables, fruits and nuts, legumes, fish, and cereal, 0 is allocated to people whose consumption is below the median value and 1 to those whose consumption is at or above the median value. For components that are assumed to be harmful to health, including meat, dairy products, and poultry, those who consume less than the median value are allocated a value of 1 , and those who consume more are allocated a value of 0 . In the case of ethanol, a value of 1 is allocated to men whose consumption is between 10 and $50 \mathrm{~g}$ per day and to women whose consumption is 5 to $25 \mathrm{~g}$ per day. The aggregate MDS ranges from 0 , indicating minimum adherence to the MDP, to 9 as maximum adherence.

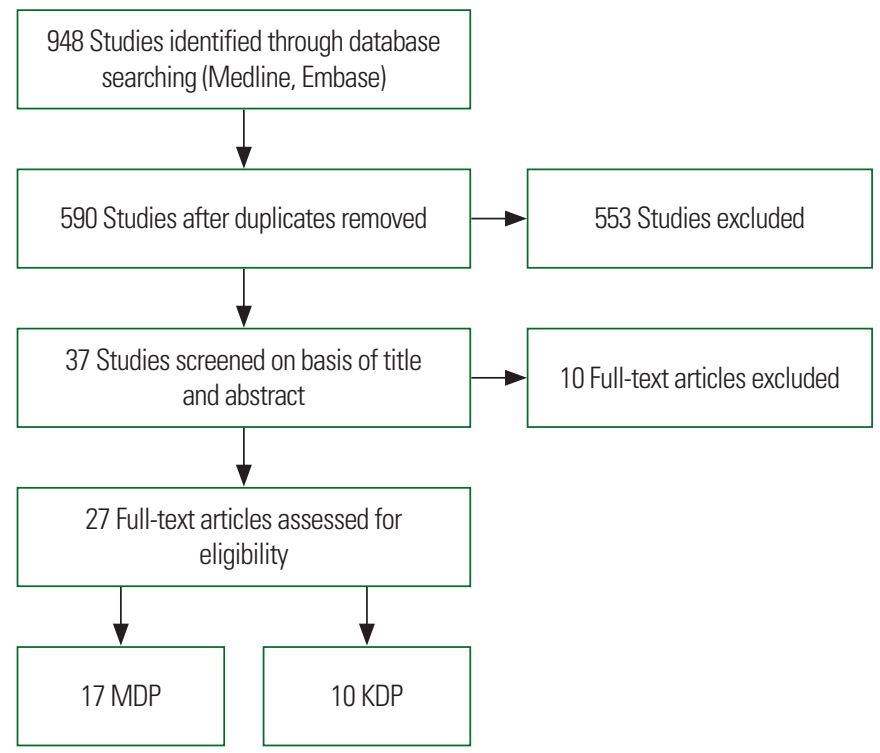

Figure 1. Study selection process. MDP, Mediterranean dietary pattern; KDP, Korean dietary pattern.

\section{THE KDP AND OBESITY}

We selected 10 cross-sectional studies aiming to identify an association between the KDP and obesity for this review (Table 1). There were some differences in the main components of the KDP through dietary pattern analysis; however, the food items with the highest factor loading of the KDP were white rice, kimchi, vegetables, and fish in most studies. In a study by Shin et al. ${ }^{18}$, the group with the highest traditional dietary pattern score showed a $24 \%$ decreased risk of abdominal obesity compared with the group with the lowest score (quintile 5 [Q5] vs. Q1: OR, 0.76; 95\% CI, 0.590.98). Three articles showed no significant association between the KDP and obesity. ${ }^{19,20,51} \mathrm{Kim}$ et $\mathrm{al}^{21}{ }^{21}$ and Choi et al. ${ }^{22}$ reported that the group with the highest KDP score had an increased likelihood of abdominal obesity (Q5 vs. Q1: OR, 1.07; 95\% CI, 1.01-1.16; Q5 vs. Q1: OR, 1.27; 95\% CI, 1.02-1.57). In a study by Kim et $\mathrm{al}^{52}$, the highest tertile of the KDP score showed an increased risk of obesity compared to the lowest tertile (tertile 3 [T3] vs. T1: OR, 1.19; 95\% CI, 1.06-1.33; $P$ for trend $=0.004)$. Three studies using cluster analysis compared prevalence of obesity between the KDP group and the WDP group. ${ }^{20,53,54}$ The WDP group had $80 \%$ higher risk of abdominal obesity than the KDP group (WDP vs. KDP: OR, 1.81; 95\% CI, 1.10-2.98) $)^{53}$ and 40\% higher risk of obesity diagnosed using BMI than the KDP group (KDP vs. WDP: OR, 1.40; 95\% CI, 1.00-1.97)..$^{20}$ In the study by Choi et al..$^{54}$, the OR for obesity was not considerably different between the KDP and WDP.

\section{THE MDP AND OBESITY}

Table 2 shows an overview of the selected studies $(n=17)$, which include 12 cross-sectional ${ }^{23-34}$ and five prospective cohort studies. ${ }^{35-39}$ Except for two studies ${ }^{31,32}$ using factor analysis, all studies used scoring methods to identify adherence to MDP. Of the 17 articles, 12 reported that the MDP has a preventive effect on obesity. ${ }^{24-33,35,37}$ Eight cross-sectional articles showed that high MDP adherence was associated with lower risk of obesity in both men and women. ${ }^{24-26,29-33}$ Of the 12 cross-sectional studies, two confirmed a significant inverse association of adherence to the MDP with obesity in women $(\mathrm{OR}, 0.79$; $95 \% \mathrm{CI}, 0.65-0.96$; high vs. low score: 
Table 1. Korean dietary pattern and obesity (cross-sectional study, $n=10$ )

\begin{tabular}{|c|c|c|c|c|c|}
\hline Author (year) & Study design & Subject & Dietary pattern & Outcome & Effect size (95\% Cl) \\
\hline Kim et al. $(2012)^{52}$ & Cross-sectional & $\begin{array}{l}10,089 \text { Adults aged } \geq 19 \mathrm{yr} \\
\text { in Korea (KNHANES) }\end{array}$ & $\begin{array}{l}\text { (1) White rice and kimchi } \\
\text { (2) High-fat, sweets, and coffee } \\
\text { (3) Meat and alcohol } \\
\text { (4) Grains, vegetables, and fish }\end{array}$ & Obesity (BMI $\geq 25 \mathrm{~kg} / \mathrm{m}^{2}$ ) & $\begin{array}{l}\text { (1) OR, } 1.19(1.06-1.33) \text {; } \\
\text { Pfor trend }=0.004\end{array}$ \\
\hline Kim et al. $(2012)^{21}$ & Cross-sectional & $\begin{array}{l}\text { 3,742 Women aged 40-69 yr } \\
\text { in Korea (KoGES) }\end{array}$ & $\begin{array}{l}\text { (1) Animal food pattern } \\
\text { (2) Rice and vegetable pattern } \\
\text { (3) Bread and dairy pattern } \\
\text { (4) Noodle pattern }\end{array}$ & $\begin{array}{l}\text { Obesity }\left(\mathrm{BMl} \geq 25 \mathrm{~kg} / \mathrm{m}^{2}\right) \\
\text { Abdominal obesity (waist circumference } \\
\geq 80 \mathrm{~cm} \text { ) }\end{array}$ & $\begin{array}{l}\text { (1) NS for obesity } \\
\text { (2) OR, } 1.07(1.01-1.16) \text {; } \\
\text { Pfor trend <0.05 for } \\
\text { abdominal obesity }\end{array}$ \\
\hline Shin et al. $(2014)^{18}$ & Cross-sectional & $\begin{array}{l}\text { 10,711 Adults aged 19-64 yr } \\
\text { in Korea (KNHANES) }\end{array}$ & $\begin{array}{l}\text { (1) Traditional dietary pattern } \\
\text { (2) Meat and fast food pattern }\end{array}$ & $\begin{array}{l}\text { Abdominal obesity (waist circumference } \\
\geq 90 \mathrm{~cm} \text { in men and } \geq 80 \mathrm{~cm} \text { in } \\
\text { women) }\end{array}$ & $\begin{array}{l}\text { (1) } 0 \text { R, } 0.76(0.59-0.98) \text {; } \\
\text { Pfor trend }=0.07\end{array}$ \\
\hline Kim et al. $(2007)^{53}$ & Cross-sectional & $\begin{array}{l}944 \text { Adolescents aged 10-19yr } \\
\text { in Korea (KNHANES) }\end{array}$ & $\begin{array}{l}\text { (1) Traditional Korean pattern } \\
\text { (2) Western pattern } \\
\text { (3) Modified pattern }\end{array}$ & $\begin{array}{l}\text { Abdominal obesity (waist circumference } \\
\geq 90 \text { th percentile) }\end{array}$ & $\begin{array}{l}\text { (1) vs. (2) OR, } 1.81(1.10- \\
\text { 2.98) }\end{array}$ \\
\hline Choi et al. $(2015)^{22}$ & Cross-sectional & $\begin{array}{l}\text { 5,189 Women aged 31-70 yr } \\
\text { in Korea }\end{array}$ & $\begin{array}{l}\text { (1) Traditional pattern } \\
\text { (2) Western pattern } \\
\text { (3) Prudent pattern }\end{array}$ & $\begin{array}{l}\text { Abdominal obesity (waist circumference } \\
\geq 80 \mathrm{~cm} \text { ) }\end{array}$ & $\begin{array}{l}\text { (1) OR, } 1.27(1.02-1.57) \text {; } \\
\text { Pfor trend }=0.02\end{array}$ \\
\hline Song et al. $(2010)^{51}$ & Cross-sectional & $\begin{array}{l}\text { 4,347 Adolescents aged } \\
\text { 10-19 yr in Korea (KNHANES) }\end{array}$ & $\begin{array}{l}\text { (1) Traditional pattern } \\
\text { (2) Modified pattern } \\
\text { (3) Western pattern }\end{array}$ & $\begin{array}{l}\text { Obesity (BMI } \geq 95 \text { th percentile for age } \\
\text { and sex)* } \\
\text { Abdominal obesity (waist circumference } \\
\geq \text { 90th percentile for age and sex)* }\end{array}$ & $\begin{array}{l}\text { (1) NS for obesity and } \\
\text { abdominal obesity }\end{array}$ \\
\hline Hong et al. $(2012)^{55}$ & Cross-sectional & $\begin{array}{l}406 \text { Adults aged } 22-78 \text { yr in } \\
\text { Korea }\end{array}$ & $\begin{array}{l}\text { (1) Korean traditional pattern } \\
\text { (2) Alcohol and meat pattern } \\
\text { (3) Sweets and fast food pattern } \\
\text { (4) Fruit and dairy pattern }\end{array}$ & $\begin{array}{l}\text { Abdominal obesity (waist circumference } \\
\geq 80 \mathrm{~cm} \text { for women or } \geq 90 \mathrm{~cm} \text { for } \\
\text { men) }\end{array}$ & $\begin{array}{l}\text { (1) NS for abdominal } \\
\text { obesity }\end{array}$ \\
\hline Choi et al. $(2011)^{54}$ & Cross-sectional & $\begin{array}{l}284 \text { Children aged 7-8yr in } \\
\text { Korea }\end{array}$ & $\begin{array}{l}\text { (1) Korean pattern } \\
\text { (2) Modified Western pattern } \\
\text { (3) Western pattern }\end{array}$ & Obesity (obesity index $\geq 20 \%)^{\ddagger . \neq}$ & (1) vs. (3) NS for obesity \\
\hline Cho et al. $(2011)^{20}$ & Cross-sectional & $\begin{array}{l}\text { 4,984 Women aged 30-79 yr } \\
\text { in Korea }\end{array}$ & $\begin{array}{l}\text { (1) Western pattern } \\
\text { (2) Healthy pattern } \\
\text { (3) Traditional pattern }\end{array}$ & Obesity (BMI $\geq 25 \mathrm{~kg} / \mathrm{m}^{2}$ ) & (3) NS for obesity \\
\hline Lee et al. $(2011)^{19}$ & Cross-sectional & $\begin{array}{l}\text { 4,762 Men aged 40-69 yr in } \\
\text { Korea (KoGES) }\end{array}$ & $\begin{array}{l}\text { (1) Animal food pattern } \\
\text { (2) Rice and vegetable pattern } \\
\text { (3) Noodle and bread pattern }\end{array}$ & $\begin{array}{l}\text { Obesity }\left(\mathrm{BMl} \geq 30 \mathrm{~kg} / \mathrm{m}^{2} \text { ) }\right. \\
\text { Abdominal obesity (waist circumference } \\
\geq 90 \mathrm{~cm} \text { ) }\end{array}$ & $\begin{array}{l}\text { (2) NS for obesity and } \\
\text { abdominal obesity }\end{array}$ \\
\hline
\end{tabular}

*The reference values for BMl and waist circumference percentile were adapted from the Korean Pediatric Growth Chart; '0besity index (\%)=[(present weight-standard weight)/ standard weight] $\times 100$; :Standard body weight was defined as the 50th percentile value for children of the same age, sex, and height according to the 2007 Standard for Korean Children and Adolescents Growth.

Cl, confidence interval; KNHANES, Korea National Health and Nutrition Examination Survey; BMI, body mass index; OR, odds ratio; KoGES, Korean Genome and Epidemiology Study; NS, not significant.

OR, 0.13; 95\% CI, 0.06-0.24). ${ }^{25,28}$ In the study by El Rhazi et al. ${ }^{23}$, prevalence of obesity was significantly associated with decreased MDP adherence (obese vs. non-obese group: OR, 1.56; 95\% CI, 1.16-2.11). One article ${ }^{34}$ showed no significant association between MDP adherence and obesity. Among the five cohort studies $^{35-37}$, Mendez et al. ${ }^{37}$ and Zappalà et al. ${ }^{35}$ reported significant inverse association between MDP adherence and obesity (high vs. low score: OR for men, 0.68 ; 95\% CI, 0.53-0.89 and OR for women, 0.69 ; 95\% CI, 0.54-0.89 and high vs. low score: OR, 0.53; 95\% CI, 0.32-0.89). However, no association was found between MDP adherence and overweight or obesity in the other three prospective studies (Beunza et al. ${ }^{36}$, Sánchez-Villegas et al. ${ }^{38}$, and Woo et al. ${ }^{39}$ ).

\section{CONCLUSION}

In this review, counter to our hypothesis that the Korean diet has a preventive association with obesity, most studies reported no significant association between the KDP and obesity. On the other hand, there was considerable epidemiological evidence for the preventive effect of the MDP on obesity. Only one of 10 articles iden- 
Table 2. Mediterranean dietary pattern and obesity

\begin{tabular}{|c|c|c|c|c|c|}
\hline Author (year) & Study design & Subject & Diet component & Outcome & Effect size (95\% Cl) \\
\hline \multicolumn{6}{|c|}{ Cross-sectional study $(n=12)$} \\
\hline Tognon et al. $(2014)^{29}$ & Cross-sectional & $\begin{array}{l}\text { 16,220 Children aged 2-9 yr } \\
\text { in } 8 \text { European countries } \\
\text { (Sweden, Italy, Germany, } \\
\text { Spain, Hungary, Belgium, } \\
\text { Estonia, and Cyprus) }\end{array}$ & $\begin{array}{l}(+) \text { : Vegetables, legumes, fruit, } \\
\text { nuts, cereals, fish } \\
(-) \text { : Dairy, meat products }\end{array}$ & $\begin{array}{l}\text { Overweight including obesity } \\
\text { (BMl: cutoffs calculated by } \\
\text { IOTF database) }\end{array}$ & $\begin{array}{l}\text { High MD adherence: obesity } \downarrow \\
(0 \mathrm{R}, 0.85 ; 95 \% \mathrm{Cl}, 0.77-0.94)\end{array}$ \\
\hline Peng et al. $(2017)^{34}$ & Cross-sectional & $\begin{array}{l}\text { 5,268 Children aged } \\
11-19 \text { yr in Israel }\end{array}$ & $\begin{array}{l}\text { (+): Fruit or fruit juice, vegetables, } \\
\text { fish, pulses, cereals or grains, } \\
\text { dairy products (yoghurt, milk, } \\
\text { etc.), olive oil, nuts } \\
\text { (-): Fast food, no breakfast, baked } \\
\text { goods or pastries, sweets and } \\
\text { candy }\end{array}$ & $\begin{array}{l}\text { Age- and sex-specific cutoff } \\
\text { values: overweight (85th to } \\
\text { 95th percentile), obese } \\
\text { (>95th percentile) }\end{array}$ & $\begin{array}{l}\text { Low MD adherence: NS for } \\
\text { overweight (OR, 1.07; 95\% Cl, } \\
\text { 0.75-1.52), obese (OR, 1.15; } \\
\text { 95\% Cl, 0.72-1.84) }\end{array}$ \\
\hline Bertoli et al. $(2015)^{33}$ & Cross-sectional & $\begin{array}{l}\text { 4,388 Adults aged 18-80 yr } \\
\text { in Italy }\end{array}$ & $\begin{array}{l}\text { (+): Olive oil, vegetables, fruits, } \\
\text { wine, legumes, fish/seafood, } \\
\text { nuts, sofrito sauce } \\
\text { (-): Red or processed meat, butter, } \\
\text { cream, or margarine, soda, } \\
\text { sweets and confectionery }\end{array}$ & Obese $\left(\mathrm{BMl} \geq 30 \mathrm{~kg} / \mathrm{m}^{2}\right)$ & $\begin{array}{l}\text { High MD adherence: obesity } \downarrow \\
(\mathrm{OR}, 0.72 ; 95 \% \mathrm{Cl}, 0.56-0.92)\end{array}$ \\
\hline $\begin{array}{l}\text { Sánchez-Taínta et al. } \\
(2008)^{25}\end{array}$ & Cross-sectional & $\begin{array}{l}\text { 3,204 Adults who have } \\
\text { diabetes or } 3 \text { or more } \\
\text { other CVD risk factors } \\
\text { aged 55-80 yr in Spain }\end{array}$ & $\begin{array}{l}\text { (+): Olive oil, vegetables, fruit, } \\
\text { wine, legumes, fish or shellfish, } \\
\text { nuts, poultry, sofrito sauce } \\
\text { (-): Red meat, hamburger, meat } \\
\text { products, butter, margarine, } \\
\text { cream, sweet or carbonated } \\
\text { beverages, sweets or pastries }\end{array}$ & Obese $\left(\mathrm{BMI} \geq 30 \mathrm{~kg} / \mathrm{m}^{2}\right)$ & $\begin{array}{l}\text { High MD adherence: obesity } \downarrow \\
\quad(\mathrm{OR}, 0.81 ; 95 \% \mathrm{Cl}, 0.71-0.94)\end{array}$ \\
\hline Schröder et al. (2004) $)^{30}$ & Cross-sectional & $\begin{array}{l}\text { 3,162 Adults aged 25-74 yr } \\
\text { in Spain }\end{array}$ & $\begin{array}{l}\text { (+): Cereals, fruits, vegetables, } \\
\text { legumes, fish, nuts } \\
(-) \text { : Meat, high-fat dairy products } \\
\text { (+m): Red wine }\end{array}$ & Obese $\left(\mathrm{BMl} \geq 30 \mathrm{~kg} / \mathrm{m}^{2}\right)$ & $\begin{array}{l}\text { High MD adherence: obesity } \downarrow \\
\text { (men: OR, 0.61; } 95 \% \mathrm{Cl}, 0.40- \\
\text { 0.92; women: OR, } 0.61 ; 95 \% \\
\text { Cl, } 0.40-0.93 \text { ) }\end{array}$ \\
\hline Grosso et al. $(2014)^{28}$ & Cross-sectional & $\begin{array}{l}\text { 3,090 Adults aged >18 yr } \\
\text { in Italy }\end{array}$ & $\begin{array}{l}\text { (+): Non-refined cereals, potatoes, } \\
\text { fruit, vegetables, legumes, fish, } \\
\text { olive oil use in daily cooking } \\
(-) \text { : Meat and meat products, } \\
\text { poultry and full-fat dairy } \\
\text { products }\end{array}$ & Obese $\left(\mathrm{BMI} \geq 30 \mathrm{~kg} / \mathrm{m}^{2}\right)$ & $\begin{array}{l}\text { High MD adherence: obesity } \downarrow \\
\text { (OR, 0.35; } 95 \% \mathrm{Cl}, 0.24-0.51)\end{array}$ \\
\hline El Rhazi et al. $(2012)^{23}$ & Cross-sectional & $\begin{array}{l}\text { 2,183 Adults aged }>18 \mathrm{yr} \\
\text { in Morocco }\end{array}$ & $\begin{array}{l}\text { (+): Vegetables, legumes, fresh } \\
\text { fruits, cereals, fish, olive oil } \\
(-) \text { : Red meat, dairy products } \\
\text { (+m): Alcohol }\end{array}$ & Obesity (BMl $\geq 30 \mathrm{~kg} / \mathrm{m}^{2}$ ) & $\begin{array}{r}\text { Obesity: low adherence of } \mathrm{MD} \uparrow \\
(\mathrm{OR}, 1.56 ; 95 \% \mathrm{Cl}, 1.16-2.11)\end{array}$ \\
\hline Mistretta et al. $(2017)^{26}$ & Cross-sectional & $\begin{array}{l}\text { 1,643 Adolescents aged } \\
11-16 \text { yr in Italy }\end{array}$ & $\begin{array}{l}\text { (+): Fruit, vegetables, fish, pulses, } \\
\text { pasta or rice, cereals or grains, } \\
\text { nuts, olive oil, dairy products, } \\
\text { yoghurts or cheese } \\
(-) \text { : Fast food, no breakfast, } \\
\text { sweets and candy }\end{array}$ & $\begin{array}{l}\text { Obesity (age- and sex-specific } \\
\text { cutoff calculated by IOTF } \\
\text { database) }\end{array}$ & $\begin{array}{l}\text { High } \mathrm{MD} \text { adherence: obesity } \downarrow \\
\text { (OR, } 0.70 ; 95 \% \mathrm{Cl}, 0.56-0.87 \text { ) } \\
\text { in both boys and girls }\end{array}$ \\
\hline $\begin{array}{l}\text { Panagiotakos et al. } \\
(2006)^{27}\end{array}$ & Cross-sectional & $\begin{array}{l}\text { 1,514 Men aged 18-87 yr } \\
\text { and 1,528 women aged } \\
\text { 18-89 yr in Greece }\end{array}$ & $\begin{array}{l}\text { (+): Non-refined cereals and } \\
\text { products, fruits, vegetables, } \\
\text { olive oil, non-fat or low-fat dairy } \\
\text { products, fish, poultry, potatoes, } \\
\text { olives, pulses, nuts } \\
(-) \text { : Red meat, meat products, } \\
\text { alcohol } \\
\text { (+m): Wine, fat, eggs, sweets }\end{array}$ & Obesity (BMl > $29.9 \mathrm{~kg} / \mathrm{m}^{2}$ ) & $\begin{array}{l}\text { High MD adherence: obesity } \downarrow \\
\text { (OR, 0.49; } 95 \% \mathrm{Cl}, 0.42-0.56)\end{array}$ \\
\hline
\end{tabular}

(Continued to the next page) 
Table 2. Continued

\begin{tabular}{|c|c|c|c|c|c|}
\hline Author (year) & Study design & Subject & Diet component & Outcome & Effect size (95\% Cl) \\
\hline $\begin{array}{l}\text { Bahreini Esfahani et al. } \\
(2016)^{31}\end{array}$ & Cross-sectional & $\begin{array}{l}840 \text { Students aged } \\
15-17 \text { yr in Iran }\end{array}$ & $\begin{array}{l}\text { (1) Western pattern } \\
\text { (2) Low protein } \\
\text { (3) Asian pattern } \\
\text { (4) Salty pattern } \\
\text { (5) Sweet pattern } \\
\text { (6) Traditional pattern } \\
\text { (7) Mediterranean pattern }\end{array}$ & $\begin{array}{l}\text { Overweight: } \geq 85 \text { th percentile } \\
\text { Obesity: } \geq 95 \text { th percentile }\end{array}$ & $\begin{array}{l}\text { High MD adherence: } \\
\text { overweight } \downarrow \text { (OR, 0.50; 95\% } \\
\text { Cl, 0.27-0.73), obesity } \downarrow \text { (OR, } \\
\text { 0.48; } 95 \% \mathrm{Cl}, 0.15-0.80 \text { ) }\end{array}$ \\
\hline $\begin{array}{l}\text { Panagiotakos et al. } \\
(2007)^{24}\end{array}$ & Cross-sectional & $\begin{array}{l}188 \text { Elderly aged } \geq 65 \mathrm{yr} \\
\text { in Cyprus }\end{array}$ & $\begin{array}{l}\text { (+): Non-refined cereals, vegetables, } \\
\text { fruits, olive oil, dairy products, } \\
\text { fish, poultry, pulses, nuts, } \\
\text { potatoes, eggs, sweets } \\
\text { (-): Red meat, meat products }\end{array}$ & Obesity $\left(\mathrm{BMl}>29.9 \mathrm{~kg} / \mathrm{m}^{2}\right)$ & $\begin{array}{l}\text { High MD adherence: obesity } \downarrow \\
\text { (OR, 0.88; } P=0.001 \text { ) }\end{array}$ \\
\hline Moreira et al. $(2014)^{32}$ & Cross-sectional & $\begin{array}{l}126 \text { Elderly aged } \geq 60 \mathrm{yr} \\
\text { in Brazil }\end{array}$ & $\begin{array}{l}\text { (1) Prudent (fruit, vegetables, and } \\
\text { meat) } \\
\text { (2) Sweets and fats (pastries, sugary } \\
\text { foods, fatty foods, whole milk) } \\
\text { (3) Typical Brazilian (fried eggs, } \\
\text { cooked beans, beef, candy, } \\
\text { string beans, fried cassava) } \\
\text { (4) Mediterranean (fruit, } \\
\text { vegetables, olive oil, and nuts) } \\
\text { (5) Traditional meal (rice and } \\
\text { beans) }\end{array}$ & Overweight $\left(\mathrm{BMl} \geq 28 \mathrm{~kg} / \mathrm{m}^{2}\right)$ & $\begin{array}{l}\text { High MD adherence: } \\
\text { overweight } \downarrow \text { (OR, 0.33; 95\% } \\
\text { Cl, 0.13-0.83) }\end{array}$ \\
\hline \multicolumn{6}{|l|}{ Cohort study $(n=5)$} \\
\hline Mendez et al. (2006) & Cohort study & $\begin{array}{r}27,827 \text { Adults aged } \\
29-65 \text { yr in Spain }\end{array}$ & $\begin{array}{l}\text { (+): Fish, vegetables, fruits, } \\
\text { legumes, cereals, ratio of MFA } \\
\text { to SFA } \\
\text { (-): Meat } \\
\text { (+m): Ethanol, dairy products }\end{array}$ & Obesity (BMI $\geq 30 \mathrm{~kg} / \mathrm{m}^{2}$ ) & $\begin{array}{l}\text { High MD adherence: obesity } \downarrow \\
\text { (men: OR, 0.68; 95\% Cl, } \\
\text { 0.53-0.89; women: OR, 0.69; } \\
\text { 95\% Cl, 0.54-0.89), NS for } \\
\text { overweight (men: OR, 1.11; } \\
\text { 95\% Cl, 0.81-1.52; women: } \\
\text { OR, 0.99; 95\% Cl, 0.78-1.25) }\end{array}$ \\
\hline Beunza et al. $(2010)^{36}$ & Cohort study & $\begin{array}{l}\text { 10,376 Adults with an } \\
\text { average age of } 38 \mathrm{yr} \\
\text { in Spain }\end{array}$ & $\begin{array}{l}\text { (+): Vegetables, fruit and nuts, } \\
\text { legumes, cereals, bread and } \\
\text { potatoes, ratio of monounsaturated } \\
\text { fatty acids to saturated fatty } \\
\text { acids, moderate alcohol } \\
\text { (-): Meat and poultry, dairy products }\end{array}$ & $\begin{array}{l}\text { Overweight or obesity } \\
\left.\text { (BMl } \geq 25 \mathrm{~kg} / \mathrm{m}^{2}\right)\end{array}$ & $\begin{array}{l}\text { High MD adherence: NS for } \\
\text { overweight or obesity (hazard } \\
\text { ratio, 0.90; 95\% Cl, 0.75-1.06) }\end{array}$ \\
\hline Zappalà et al. (2018) & Cohort study & $\begin{array}{l}2,044 \text { Adults aged } \geq 18 \mathrm{yr} \\
\text { in Italy }\end{array}$ & $\begin{array}{l}\text { (+): Fruit, vegetables, cereals, } \\
\text { legumes, fish } \\
(-) \text { : Meat and meat products, } \\
\text { dairy products } \\
\text { (+m): Alcohol }\end{array}$ & $\begin{array}{l}\text { Overweight } \\
\left(\mathrm{BMI} 25-29.9 \mathrm{~kg} / \mathrm{m}^{2}\right) \text {, obese } \\
\left(\mathrm{BMl}>30 \mathrm{~kg} / \mathrm{m}^{2}\right)\end{array}$ & $\begin{array}{l}\text { High MD adherence: obesity } \downarrow \\
\text { (OR, 0.53; } 95 \% \mathrm{Cl}, 0.32-0.89)\end{array}$ \\
\hline $\begin{array}{l}\text { Sánchez-Villegas et al. } \\
(2006)^{38}\end{array}$ & Cohort study & $\begin{array}{l}\text { 6,319 Adults (university } \\
\text { graduates) in Spain }\end{array}$ & $\begin{array}{l}\text { (+): Cereals, vegetables, fruits, } \\
\text { legumes, fish, nuts, olive oil } \\
(-) \text { : Meat/meat products, } \\
\text { whole-fat dairy products } \\
\text { (+m): Red wine }\end{array}$ & $\begin{array}{l}\text { Overweight or obesity } \\
\qquad\left(\mathrm{BMl} \geq 25 \mathrm{~kg} / \mathrm{m}^{2}\right)\end{array}$ & $\begin{array}{l}\text { High MD adherence: NS for } \\
\text { overweight or obesity (OR, } \\
\text { 0.90; 95\% Cl, 0.59-1.38) }\end{array}$ \\
\hline Woo et al. $(2008)^{39}$ & Cohort study & $\begin{array}{l}\text { 1,010 Adults aged } 25-74 \mathrm{yr} \\
\text { in China }\end{array}$ & $\begin{array}{l}\text { (+): Cereals, vegetables, fruit and } \\
\text { nuts, legumes, fish } \\
(-) \text { : Meat, poultry, dairy products } \\
(\mathrm{m+}) \text { : Ethanol }\end{array}$ & $\begin{array}{c}\text { Overweight }\left(\mathrm{BMI} \geq 23 \mathrm{~kg} / \mathrm{m}^{2}\right) \text {, } \\
\text { obese }\left(\mathrm{BMI}>30 \mathrm{~kg} / \mathrm{m}^{2}\right)\end{array}$ & $\begin{array}{l}\text { High MD adherence: NS for } \\
\text { overweight (OR, 1.35; 95\% Cl, } \\
\text { 0.94-1.93) }\end{array}$ \\
\hline
\end{tabular}

$\mathrm{Cl}$, confidence interval; +, positive components; -, negative components; +m, components positive in moderation; BMl, body mass index; IOTF, International Obesity Task Force; MD, Mediterranean diet; OR, odds ratio; NS, not significant; CVD, cardiovascular disease; MFA, monounsaturated fatty acid; SFA, saturated fatty acid. 
tified a significant inverse association between the KDP and obesity, whereas 12 of $17 \mathrm{MDP}$ studies reported a preventive association between the MDP and obesity.

Although the KDP and MDP are both considered healthy diets ${ }^{23,27,52}$, the differences between them in our results might be attributable to differences in the detailed dietary components. Adherence to the MDP leads to adequate intake of various nutrients like vitamin $\mathrm{C}$, tocopherols, carotene, linolenic acid, and minerals and that of beneficial non-nutrients like anthocyanins and polyphenols. ${ }^{27}$ Compared to the MDP, the typical KDP generally lacks fruit and dairy products and is high in sodium. Thus, for the KDP to provide greater health benefits in lowering the risk of chronic diseases and to have similar effects as that of the Mediterranean diet, it may be necessary to complement it with consumption of fruit and low-fat dairy products and reduction in sodium. ${ }^{56}$ Moreover, the results of research on the KDP and MDP are different because the methods to derive them are not the same. For MDP studies, we identified the association between obesity and dietary variation based on criteria for a predefined MDP regardless of population, whereas the KDP was defined based on distinct foods or food groups (such as white rice and kimchi) with significant variation in consumption between populations. Therefore, the KDP was slightly different in each study depending on the target population. By defining the characteristics of the KDP and developing criteria for it such as for the MDS, it may be possible to evaluate the health effects of the KDP more accurately and objectively.

Results of the KDP studies were inconsistent. Differences in general characteristics of subjects may have contributed to the inconsistency of these results. Four studies ${ }^{18,22,52,55}$ were conducted on adults, two studies ${ }^{20,21}$ focused on women, two studies ${ }^{51,53}$ on adolescents, one study ${ }^{54}$ on children, and one study ${ }^{19}$ on men. Furthermore, differences in general characteristics such as income, education level, physical activity, and smoking status may also cause inconsistency in the results as confounding factors, although these factors were statistically adjusted in each study. For instance, in one $\mathrm{KDP}$ study ${ }^{18}$, the subjects within the highest quintile of the KDP were older, had more physical activity, had higher education and income levels, and were less likely to be current smokers. In the statistical analysis, the logistic regression model was adjusted for age, household income, education, smoking, physical activity, etc. In the study by Kim et al. ${ }^{21}$, those in the highest quintile of the KDP were older and had higher education and income levels, and these factors were adjusted statistically. Ours is the first study to review the association between obesity and the KDP compared with the MDP.

However, this study has some limitations. First, selected studies on the association between the KDP and obesity were mainly based on cross-sectional design, making it difficult to identify the causal association between the two factors. Second, the statistical technique of dietary pattern analysis for the KDP is somewhat subjective because food categorization, labeling of components, and extracting the number of factors can be arbitrary. Due to the subjective aspect of the method for deriving dietary patterns, it should be carefully applied to the general population. Third, there was a difference in diet survey method between the MDP and KDP. Dietary data were collected via the validated food-frequency questionnaire (FFQ) for all the MDP studies, while five KDP studies collected dietary information through 24-hour recall and five through the FFQ. Also, the study populations for KDP and MDP research are different. The KDP studies targeted Koreans, and the MDP studies selected Westerners, except one study that focused on the Chinese population. ${ }^{39}$ Finally, the methods to derive the KDP or MDP were different. Factor analysis explores what explains the variety in diets and how well those variations are related to risk, whereas index analysis asks whether variety from a predefined diet is related to risk. ${ }^{57}$

In this study, we only examined the association of the KDP and the MDP with obesity; further studies need to identify the association between the KDP and other chronic diseases. Well-designed controlled trials and prospective cohort studies are also needed to conclude whether the KDP is related with obesity.

\section{CONFLICTS OF INTEREST}

The authors declare no conflict of interest.

\section{REFERENCES}

1. Burton BT, Foster WR. Health implications of obesity: an NIH Consensus Development Conference. J Am Diet Assoc 1985; 
$85: 1117-21$

2. Kenchaiah S, Evans JC, Levy D, Wilson PW, Benjamin EJ, Larson MG, et al. Obesity and the risk of heart failure. N Engl J Med 2002;347:305-13.

3. Must A, Spadano J, Coakley EH, Field AE, Colditz G, Dietz $\mathrm{WH}$. The disease burden associated with overweight and obesity. JAMA 1999;282:1523-9.

4. Korea Centers for Disease Control and Prevention. National health statistics. Cheongju: Korea Centers for Disease Control and Prevention; 2016.

5. Statistics Korea. Cause of death. Daejeon: Statistics Korea; 2017.

6. World Health Organization. Fact sheets: obesity and overweight. Key facts. Geneva: World Health Organization; 2018.

7. Flynn MA, McNeil DA, Maloff B, Mutasingwa D, Wu M, Ford $\mathrm{C}$, et al. Reducing obesity and related chronic disease risk in children and youth: a synthesis of evidence with 'best practice' recommendations. Obes Rev 2006;7 Suppl 1:7-66.

8. Moreno LA, Rodríguez G. Dietary risk factors for development of childhood obesity. Curr Opin Clin Nutr Metab Care 2007; 10:336-41.

9. Weinsier RL, Hunter GR, Heini AF, Goran MI, Sell SM. The etiology of obesity: relative contribution of metabolic factors, diet, and physical activity. Am J Med 1998;105:145-50.

10. US Department of Health and Human Services, US Department of Agriculture. 2015-2020 Dietary guidelines for Americans. 8th ed. Washington (DC): US Department of Agriculture; 2015.

11. Schulze MB, Martínez-González MA, Fung TT, Lichtenstein AH, Forouhi NG. Food based dietary patterns and chronic disease prevention. BMJ 2018;361:k2396.

12.Jannasch F, Kröger J, Schulze MB. Dietary patterns and type 2 diabetes: a systematic literature review and meta-analysis of prospective studies. J Nutr 2017;147:1174-82.

13. Sofi F, Macchi C, Abbate R, Gensini GF, Casini A. Mediterranean diet and health status: an updated meta-analysis and a proposal for a literature-based adherence score. Public Health Nutr 2014;17:2769-82.

14. Appel LJ, Moore TJ, Obarzanek E, Vollmer WM, Svetkey LP, Sacks FM, et al. A clinical trial of the effects of dietary patterns on blood pressure. N Engl J Med 1997;336:1117-24.
15. Fung TT, Chiuve SE, McCullough ML, Rexrode KM, Logroscino G, Hu FB. Adherence to a DASH-style diet and risk of coronary heart disease and stroke in women. Arch Intern Med 2008;168:713-20.

16. Guenther PM, Casavale KO, Reedy J, Kirkpatrick SI, Hiza HA, Kuczynski KJ, et al. Update of the Healthy Eating Index: HEI2010. J Acad Nutr Diet 2013;113:569-80.

17. Schwingshackl L, Hoffmann G. Diet quality as assessed by the Healthy Eating Index, the Alternate Healthy Eating Index, the Dietary Approaches to Stop Hypertension score, and health outcomes: a systematic review and meta-analysis of cohort studies. J Acad Nutr Diet 2015;115:780-800.

18. Shin HJ, Cho E, Lee HJ, Fung TT, Rimm E, Rosner B, et al. Instant noodle intake and dietary patterns are associated with distinct cardiometabolic risk factors in Korea. J Nutr 2014;144: 1247-55.

19. Lee JE, Kim JH, Son SJ, Ahn Y, Lee J, Park C, et al. Dietary pattern classifications with nutrient intake and health-risk factors in Korean men. Nutrition 2011;27:26-33.

20. Cho YA, Kim J, Cho ER, Shin A. Dietary patterns and the prevalence of metabolic syndrome in Korean women. Nutr Metab Cardiovasc Dis 2011;21:893-900.

21. Kim JH, Lee JE, Jung IK. Dietary pattern classifications and the association with general obesity and abdominal obesity in Korean women. J Acad Nutr Diet 2012;112:1550-9.

22. Choi JH, Woo HD, Lee JH, Kim J. Dietary patterns and risk for metabolic syndrome in Korean women: a cross-sectional study. Medicine 2015;94:e1424.

23. El Rhazi K, Nejjari C, Romaguera D, Feart C, Obtel M, Zidouh A, et al. Adherence to a Mediterranean diet in Morocco and its correlates: cross-sectional analysis of a sample of the adult Moroccan population. BMC Public Health 2012;12:345.

24. Panagiotakos DB, Polystipioti A, Papairakleous N, Polychronopoulos E. Long-term adoption of a Mediterranean diet is associated with a better health status in elderly people; a crosssectional survey in Cyprus. Asia Pac J Clin Nutr 2007;16:331-7. 25. Sánchez-Taínta A, Estruch R, Bulló M, Corella D, GómezGracia E, Fiol M, et al. Adherence to a Mediterranean-type diet and reduced prevalence of clustered cardiovascular risk factors in a cohort of 3,204 high-risk patients. Eur J Cardio- 
vasc Prev Rehabil 2008;15:589-93.

26. Mistretta A, Marventano S, Antoci M, Cagnetti A, Giogianni G, Nolfo F, et al. Mediterranean diet adherence and body composition among Southern Italian adolescents. Obes Res Clin Pract 2017;11:215-26.

27. Panagiotakos DB, Chrysohoou C, Pitsavos C, Stefanadis C. Association between the prevalence of obesity and adherence to the Mediterranean diet: the ATTICA study. Nutrition 2006; 22:449-56.

28. Grosso G, Pajak A, Mistretta A, Marventano S, Raciti T, Buscemi S, et al. Protective role of the Mediterranean diet on several cardiovascular risk factors: evidence from Sicily, southern Italy. Nutr Metab Cardiovasc Dis 2014;24:370-7.

29. Tognon G, Hebestreit A, Lanfer A, Moreno LA, Pala V, Siani A, et al. Mediterranean diet, overweight and body composition in children from eight European countries: cross-sectional and prospective results from the IDEFICS study. Nutr Metab Cardiovasc Dis 2014;24:205-13.

30. Schröder H, Marrugat J, Vila J, Covas MI, Elosua R. Adherence to the traditional Mediterranean diet is inversely associated with body mass index and obesity in a Spanish population. J Nutr 2004;134:3355-61.

31. Bahreini Esfahani N, Ganjali Dashti N, Ganjali Dashti M, Noorv MI, Koon PB, Talib RA, et al. Dietary predictors of overweight and obesity in Iranian adolescents. Iran Red Crescent Med J 2016;18:e25569.

32. Moreira PL, Corrente JE, Boas PJ, Ferreira AL. Dietary patterns are associated with general and central obesity inelderly living in a Brazilian city. Rev Assoc Med Bras 2014;60:457-64.

33. Bertoli S, Leone A, Vignati L, Bedogni G, Martínez-González MÁ, Bes-Rastrollo M, et al. Adherence to the Mediterranean diet is inversely associated with visceral abdominal tissue in Caucasian subjects. Clin Nutr 2015;34:1266-72.

34. Peng W, Goldsmith R, Berry EM. Demographic and lifestyle factors associated with adherence to the Mediterranean diet in relation to overweight/obesity among Israeli adolescents: findings from the Mabat Israeli national youth health and nutrition survey. Public Health Nutr 2017;20:883-92.

35.Zappalà G, Buscemi S, Mulè S, La Verde M, D’Urso M, Corleo D, et al. High adherence to Mediterranean diet, but not in- dividual foods or nutrients, is associated with lower likelihood of being obese in a Mediterranean cohort. Eat Weight Disord 2018;23:605-14.

36. Beunza JJ, Toledo E, Hu FB, Bes-Rastrollo M, Serrano-Martínez M, Sánchez-Villegas A, et al. Adherence to the Mediterranean diet, long-term weight change, and incident overweight or obesity: the Seguimiento Universidad de Navarra (SUN) cohort. Am J Clin Nutr 2010;92:1484-93.

37. Mendez MA, Popkin BM, Jakszyn P, Berenguer A, Tormo MJ, Sanchéz MJ, et al. Adherence to a Mediterranean diet is associated with reduced 3-year incidence of obesity. J Nutr 2006; 136:2934-8.

38. Sánchez-Villegas A, Bes-Rastrollo M, Martínez-González MA, Serra-Majem L. Adherence to a Mediterranean dietary pattern and weight gain in a follow-up study: the SUN cohort. Int J Obes 2006;30:350-8.

39. Woo J, Cheung B, Ho S, Sham A, Lam TH. Influence of dietary pattern on the development of overweight in a Chinese population. Eur J Clin Nutr 2008;62:480-7.

40. Korea Food Research Institute. Development of Korean foods marketing model. Seongnam: Korea Food Research Institute; 2008.

41. Rural Development Administration, National Institute of Agricultural Sciences. Hansik and health. Paju: Gyomoon; 2010.

42. Woo HD, Shin A, Kim J. Dietary patterns of Korean adults and the prevalence of metabolic syndrome: a cross-sectional study. PLoS One 2014;9:e111593.

43. Popkin BM, Gordon-Larsen P. The nutrition transition: worldwide obesity dynamics and their determinants. Int J Obes Relat Metab Disord 2004;28 Suppl 3:S2-9.

44. Song Y, Joung H. A traditional Korean dietary pattern and metabolic syndrome abnormalities. Nutr Metab Cardiovasc Dis 2012;22:456-62.

45. Jun S, Ha K, Chung S, Joung H. Meat and milk intake in the rice-based Korean diet: impact on cancer and metabolic syndrome. Proc Nutr Soc 2016;75:374-84.

46. Patra JK, Das G, Paramithiotis S, Shin HS. Kimchi and other widely consumed traditional fermented foods of Korea: a review. Front Microbiol 2016;7:1493.

47. Shin D, Jeong D. Korean traditional fermented soybean prod- 
ucts: Jang. J Ethn Food 2015;2:2-7.

48. Keys A. Mediterranean diet and public health: personal reflections. Am J Clin Nutr 1995;61(6 Suppl):1321S-1323S.

49. Sofi F, Abbate R, Gensini GF, Casini A. Accruing evidence on benefits of adherence to the Mediterranean diet on health: an updated systematic review and meta-analysis. Am J Clin Nutr 2010;92:1189-96.

50. Trichopoulou A, Costacou T, Bamia C, Trichopoulos D. Adherence to a Mediterranean diet and survival in a Greek population. N Engl J Med 2003;348:2599-608.

51. Song Y, Park MJ, Paik HY, Joung H. Secular trends in dietary patterns and obesity-related risk factors in Korean adolescents aged 10-19 years. Int J Obes 2010;34:48-56.

52. Kim J, Jo I, Joung H. A rice-based traditional dietary pattern is associated with obesity in Korean adults. J Acad Nutr Diet 2012;112:246-53.

53. Kim JA, Kim SM, Lee JS, Oh HJ, Han JH, Song Y, et al. Dietary patterns and the metabolic syndrome in Korean adoles- cents: 2001 Korean National Health and Nutrition Survey. Diabetes Care 2007;30:1904-5.

54. Choi HJ, Joung H, Lee HJ, Jang HB, Kang JH, Song J. The influence of dietary patterns on the nutritional profile in a Korean child cohort study. Osong Public Health Res Perspect 2011;2: 59-64.

55. Hong S, Song Y, Lee KH, Lee HS, Lee M, Jee SH, et al. A fruit and dairy dietary pattern is associated with a reduced risk of metabolic syndrome. Metabolism 2012;61:883-90.

56. McAuley KA, Hopkins CM, Smith KJ, McLay RT, Williams SM, Taylor RW, et al. Comparison of high-fat and high-protein diets with a high-carbohydrate diet in insulin-resistant obese women. Diabetologia 2005;48:8-16.

57. Reedy J, Wirfält E, Flood A, Mitrou PN, Krebs-Smith SM, Kipnis V, et al. Comparing 3 dietary pattern methods-cluster analysis, factor analysis, and index analysis-with colorectal cancer risk: The NIH-AARP Diet and Health Study. Am J Epidemiol 2010;171:479-87. 